\title{
The Digital Clinic: A New Developed way for diagnosis and medication
}

\author{
Hossam Mohamed Abdelfattah Saber \\ Dakahlia STEM High School \\ Kafr Sad, Damietta, Egypt \\ Hossam.1319057@stemdakahlia.moe.edu.eg
}

Yousef Ahmed Mahmoud Sobhi

Dakahlia STEM High School

Abu Kbeer, Sharqia, Egypt

Yousef.1319029@stemdakahlia.moe.edu.eg

DOI: 10.31364/SCIRJ/v9.i03.2021.P0321847

http://dx.doi.org/10.31364/SCIRJ/v9.i03.2021.P0321847

\begin{abstract}
When it comes to health, we see an evident carelessness from individuals in their health regulation that might interrupt their daily life routine and make them prone to dangerous diseases or even death. The reasons behind this wrong behavior are simple: people do not have time to care about their health due to heavy workload. Moreover, costly, complicated checkups contributed to that action as well. Health-Related research found that approximately 2000 people died monthly because of their medical negligence. As a result, this issue must be treated with a low-cost and efficient solution. In turn, this solution has to provide a formatted environment for patients or individuals to give enough care of their health insurance. Our initial idea is to construct a highly efficient health application that helps people track and monitor their health data and give them guaranteed tips to improve their health state to avoid bad habits or myths. The visualization of data in the android application provides the user with a comprehensive conclusion that devoid of any complexity. By using an integrated network of sensors controlled via microcontrollers, it is now possible to process those data without any difficulty. This system can sense body temperature, humidity, blood pressure, glucose rate, and respiration rate.
\end{abstract}

Index Terms - JavaScript, Heart rate, Temperature sensor, Healthcare apps, Arduino. (key words)

\section{INTRODUCTION}

Over the decades, the Egyptian healthcare system faced challenges in improving and ensuring ordinary people's health states. Egyptian situation is due to the burden of combating illnesses associated with poverty and lack of education and the inefficient functioning of healthcare delivery. Shortcomings in human resources include low capacities and skills, maldistribution of physicians across geographic regions and specialties, and insufficient salaries and incentives. Additionally, the health facilities' infrastructure -building, furniture, and maintenance- is deteriorating. Accordingly, the appropriate features the solution must include are cutting out unnecessary and expensive trips to the doctors, providing the patient with a full library of medical information, and facilitating the communication between the patient and the physicians. Initially, solving those challenges is a mobile application empowered by an external device that serves as a data source for the app. The main goal behind this system is to ensure that the patient or the user, in general, is comfortable with the examination and accessibility. Our design requirements regarding the mobile app testing are simplicity during the usage, accessibility of the data and medical library of the drugs and diseases, and, finally, the efficiency, measured through if the data set provided is accurate enough. The tips are working. For further explanation, both of them will be tested by conducting surveys among concerned people to know what level they are satisfied with the whole system or the general idea. To this end, the solution correlates the benefits of using software applications with hardware devices, especially mobile applications represented in a system of interrelated computing devices, to replace the doctor's counseling in ample situations.

\section{MATERIALS}

1. Arduino Mega R3

2. Heart Rate Sensor

3. Bluetooth Module

4. Graphical LCD $(128 \times 64)$

5. Humidity Sensor DHT11

6. Waterproof Temperature Sensor DS18b20

7. TFT-3.5 inch screen

8. LEDs

9. Arduino Mega Shield

10. $\mathrm{C}++$, Java Script, HTML, and CSS 


\section{METHODS}

Especially in this project, the methods varied between hardware and software methods. Each of them required rigorous and hard work.

For the Software, we did the following (Figure 1\&2):

1 - Searching for a subtle challenge and getting the idea of the application.

2- Determining the appropriate variables (inputs like temperature and outputs like the health tips and schedules).

3- Processing the health data and comparisons between different parameters to provide the user with useful tips.

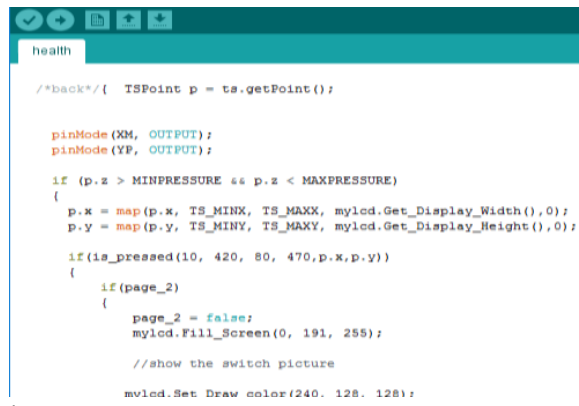

Figure 1

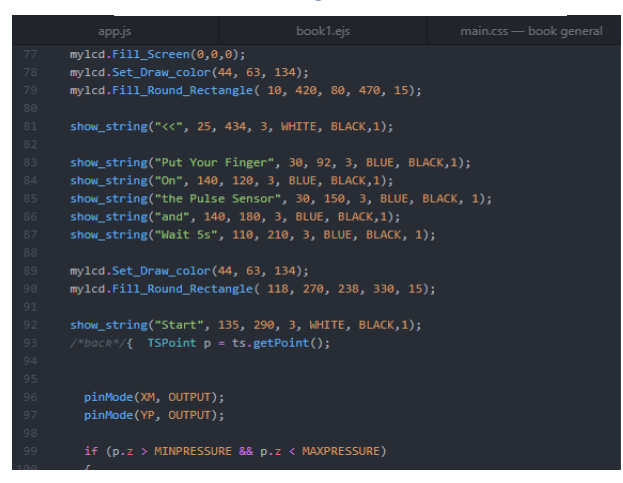

4- Programming the application with JavaScript language (hybrid app).

5- Testing the app to detect the syntax and logical errors that occurred in the code.

6- Publishing the app to be officially used by people using the Heroku server and GoNative.io services to publish the android versions.

\section{- For the hardware, we did the following (Figure} 3\&4):

1- Designing the device and provide it with useful features that are suitable for the android app.

2- Applying the design to wood with the help of a laser cutter machine from Fab Lab.

\section{3- Enhancing the} device with various

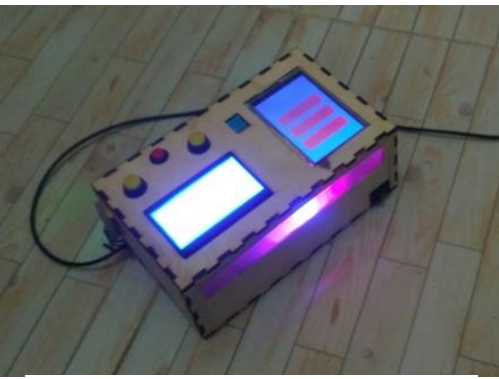
medical sensors to maintain its functionality like body temperature, respiration rate, and heart rate sensor.

4- Finalizing the prototype and ensuring the wires and sensors connection.

5- configuring the device appropriately with the android app.

Figure 4

After completing the application and the device, we are ready to test our system based on the design requirements we have put into ensuring that it is working probably without any drops in data gained or interruption in sensors.

Test-plan is essential to see whether the project will function correctly or not and be modified to achieve suitable results.

For the software test, we will first test the back end's functionality to get the quickest analysis with the least time. We were then testing the pages' back-end to eliminate logical and runtime errors. After that, we will pilot the application. We asked a group of patients to use our application once every 24 hours for a week. They have to give feedback describing our application's functionality and feasibility and describe their experience in general.

For the hardware test: Body Temperature sensor, by measuring several patients' body temperature then comparing the data with values for the same patients got by a thermometer. We can calculate the error percentage.

Heart Rate sensor, We measured several patients' heart rate relative to the accuracy and the error percentage of the values by pulse sensor concerning the EKG heart rate monitor values accuracy and error percentage.

DHT 11 sensor will compare the sensor measurements for the temperature and humidity of the surrounding air with that reported in the weather broadcast.

\section{ANALYSIS}

Our web application depends on the user's data, analyzing it to diagnose the user's health state. It provides him with health tips to help him overcome the situation.

\section{Software Part:}

Variables are the first central portion of the software, determining what the inputs are and the results provide a clear view of the application functionality: -

Inputs: the application will not work by itself; it has to consider some information to avoid any wrong instruction, which later can cause adverse consequences. These inputs are height, weight, disease history, blood type, body temperature, and heart rate.

Outputs: after getting the inputs, the application processes them and makes compressions to determine what the patient suffers from; consequently, it gives the user-health tips and provides him with antidots to help him.

\section{User-Interface:}

To construct simple and attractive interface to grape the user thought we used a limited number of buttons and input fields to avoid any distractions or complexity in addition to choose suitable colors which their hex codes are (\#8BD8BD, \#FDEBEC, \#2E3C7D). we used Hyper Text Markup Language (HTML) to build the web app structure. The following code we wrote to create the structure in the figure(6):

$<$ form class="name" action="index.html" method="post" id="name" >

$$
\begin{aligned}
& <\text { p }>\text { Enter Your Name }</ \mathrm{p}> \\
& <\text { input type="text" name="" value=""> } \\
& <\text { button type="submit" id="subName" }>\text { Next }<\text { /button }> \\
& </ \text { form }>
\end{aligned}
$$




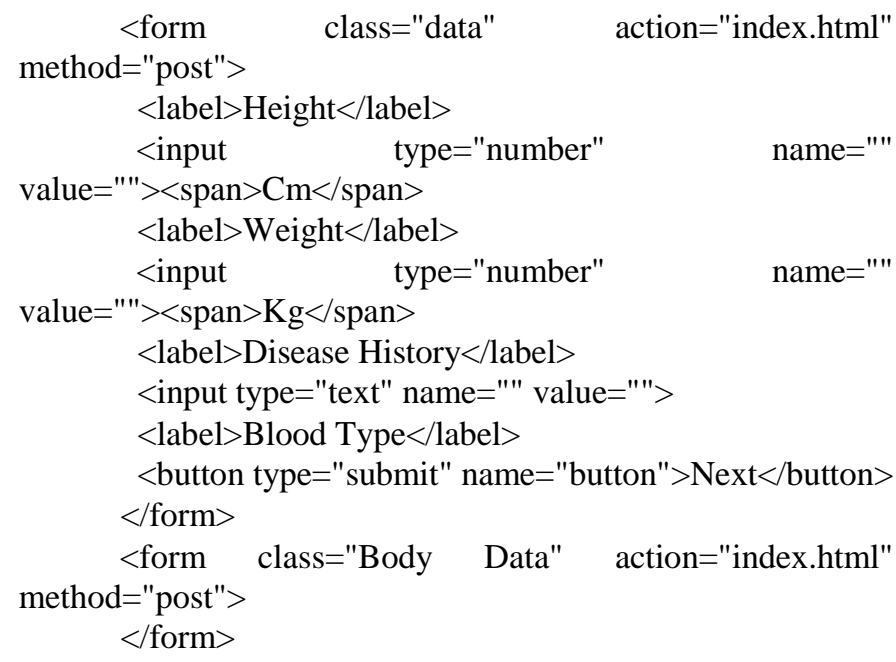

\section{Back-end:}

To code the back end of the server, which process the inputs and make comparisons and operations to get the results, we used node.js. in specific, individual packages:

- Socket.io package: this package we used to transfer the data from the server to the pages and vice versa. It, in general, creates the network.

- Body-parser: this package we used to simplify the routing of the application.

- To program the back end of the pages, we used jQuery (JavaScript library). We set a function for the button of id(\#subName), the button responsible for submitting the user's name, for moving to the next page if the user makes the event (click).

\section{Hardware Part:}

Our application correlates with a hardware device to get some numerical information the patient usually struggles with getting it. Regardless, we constructed a small box the user can use to transfer accurate data to the application to schedule. The box based on three primary sensors to get the data:

DS18B20 Sensor is a temperature-sensing device; the user uses this sensor to measure his body temperature by holding the sensor for 5 seconds; the sensor sends the measurements to the Arduino, which records a value every 200milliseconds then it takes the average. After that, the Arduino stores the results in an object called (tempVal).

- Pulse Sensor, this detector consists of a lightemitting diode and a photodiode; the amount of light absorbed depends on the tissue's blood volume. It calculates the variations in the blood flow in that tissue caused by the heartbeat pulses; the sensor can measure the patient's heart rate. Then the sensor sends the measurements to the Arduino to be saved in an object called (hrtRate).

DHT11 Sensor, an actual digital temperature and humidity sensor, can measure the temperature and humidity of the surrounding air, providing brief information about whether to be sent to the application to consider the processing step. After the Arduino gets the data and stores it in the object form, it sends it to the application through Bluetooth to be scheduled.

\section{Design requirements:}

- Accessibility: we created our application as webbased to extend the range of devices and ways to access it. As a web application, the user can install the application on his mobile phone; also, anyone who has the link can use our application.

- Simplicity: we designed our web application based on simple backend methods to reduce the routing expressed in the node.js server. Thus, we prevented the users from being distracted or confused. Also, these simple methods created a design that fits different ranges of ages, including youth and children.

\section{RESULTS}

We have collected some results regarding the application's testing, especially the conducted surveys, which were initially made to know how people's level of satisfaction towards the application and its idea in general. We tested the app among randomly- chosen twelve users of different ages and genders from around the community. It can be concluded that the application has merely achieved some of the design requirements, which have been put to the test of the app. Almost all the testers revealed great feedback and satisfaction with the new technology fast method of diagnosing and medication. Furthermore, the overall gain is considered positive. The negative responses will significantly impact encouraging us to improve the quality of the usage based on the users' collected comments according to their experience in using the app and deal with its different components and functionalities.

\section{CONCLUSION}

HealthCare Mobile applications are so beneficial in many different aspects of rescuing the patient's health state and tracking his health-based data. In our case, the mobile application is not operating lonely; instead, it is embedded with an external prototype that acts as a data provider for the mobile application; however, the application can be independent in its functions, but it would much better if the customer used the device for more accurate data measurement and easy data gaining. Our central goal behind this system (applicationdevice) is getting rid of any complexity present in any prior applications, easing the process of tracking health data, and reducing many doctors' efforts, so it will give them the chance to focus on more complicated surgeries, and other rigorous treatment. Furthermore, the mobile app will provide the user with the expected disease based on the data gained from the device, tips to reduce the symptoms of the disease, and improve the user's conditions and regulate daily and weekly schedules to examine the user's conditions. In summation, the system has successfully achieved its original purpose in approaching simplicity and higher productivity.

\section{ACKNOWLEDGMENT}

This project consumed a lot of effort, attempts, and research. Implementation would not have been possible if we did not have the support of many individuals and organizations. Therefore, we would like to extend our gratitude to all of them. After Allah, we are thankful to Ayman Mohamed, Zyad Elharairy, and Amgad Elkhsosy (Medical students). In addition to Mohamed Rezk, Khaled Fahmy, and Abdelrahman Adel as they are (Engineering students). Both of the two groups have 
helped us a lot as they pursue their studies in our project, Biomedical and Engineering.

\section{REFERENCES}

[1] Healthcare recession. Mehrem, H. K. (2015, September 24). Healthcare: A Neglected Public Good. Retrieved October 7, 2020, from https://egyptianstreets.com/2015/09/24/healthcare-aneglected-public-good/.

[2] Types of application. What Are the Different Types of Mobile Apps? (n.d.). Retrieved October 9, 2020, from https://clevertap.com/blog/types-of-mobile-apps/.
[3] Prior healthcare Mobile apps. Bazzoli, F. (2019, July 29). 20 apps for healthcare providers. Retrieved October 14, 2020, from https://www.healthdatamanagement.com/list/20-notableapps-for-provider-organizations.

[4] Patient healthcare system. Instructables. (2017, September 23). IOT Based Healthcare System. Retrieved November 2, 2020, from https://www.instructables.com/id/IOTBased-Healthcare-System-for-Elderly/.

[5] Hybrid Mobile application. Cowart, J. (2019, October 10). What is a Hybrid Mobile App? Retrieved November 4, 2020, from https://www.telerik.com/blogs/what-is-ahybrid-mobile-app-. 